\title{
Shot noise and conductivity at high bias in bilayer graphene: Signatures of electron-optical phonon coupling
}

\author{
A. Fay, ${ }^{1}$ R. Danneau, ${ }^{1,2}$ J. K. Viljas,,${ }^{1, *}$ F. Wu,,${ }^{1,2}$ M. Y. Tomi, ${ }^{1}$ J. Wengler, ${ }^{1}$ M. Wiesner, ${ }^{1,3}$ and P. J. Hakonen ${ }^{1}$ \\ ${ }^{1}$ Low Temperature Laboratory, Aalto University, P.O.Box 15100, FI-00076 AALTO, Finland \\ ${ }^{2}$ Institute of Nanotechnology and Institute of Physics, Karlsruhe Institute of Technology, Germany \\ ${ }^{3}$ Faculty of Physics, Adam Mickiewicz University, 61-614 Poznan, Poland
}

(Received 31 August 2011; revised manuscript received 25 November 2011; published 14 December 2011)

\begin{abstract}
We have studied electronic conductivity and shot noise of bilayer graphene (BLG) sheets at high bias voltages and low bath temperature $T_{0}=4.2 \mathrm{~K}$. As a function of bias, we find initially an increase of the differential conductivity, which we attribute to self-heating. At higher bias, the conductivity saturates and even decreases due to backscattering from optical phonons. The electron-phonon interactions are also responsible for the decay of the Fano factor at bias voltages $V>0.1 \mathrm{~V}$. The high bias electronic temperature has been calculated from shot-noise measurements, and it goes up to $\sim 1200 \mathrm{~K}$ at $V=0.75 \mathrm{~V}$. Using the theoretical temperature dependence of BLG conductivity, we extract an effective electron-optical phonon scattering time $\tau_{\text {e-op }}$. In a 230-nm-long BLG sample of mobility $\mu=3600 \mathrm{~cm}^{2} \mathrm{~V}^{-1} \mathrm{~s}^{-1}$, we find that $\tau_{\mathrm{e}-\mathrm{op}}$ decreases with increasing voltage and is close to the charged impurity scattering time $\tau_{\text {imp }}=60$ fs at $V=0.6 \mathrm{~V}$.
\end{abstract}

DOI: 10.1103/PhysRevB.84.245427

PACS number(s): 73.63.-b, 73.23.-b, 73.50.Fq, 73.50.Td

\section{INTRODUCTION}

Graphene, a two-dimensional plane of carbon atoms arranged in a honeycomb lattice, has recently attracted wide attention due to its unique properties. ${ }^{1}$ High electronic mobility ${ }^{2}$ up to more than $2 \times 10^{5} \mathrm{~cm}^{2} \mathrm{~V}^{-1} \mathrm{~s}^{-1}$ combined with a good scalability makes graphene-based field-effect transistors (FETs) potential basic building blocks for future nanoelectronics devices. A large on/off current ratio is required to challenge the current $\mathrm{Si}$-based FETs. In monolayer graphene (MLG), the on/off current ratio is low $(\sim 5)$ because the conductivity always remains above a value of roughly $4 e^{2} / h$ or $4 e^{2} / \pi h$ for diffusive and ballistic ${ }^{3,4}$ samples, respectively. In bilayer graphene (BLG), this ratio can amount up to 20000 thanks to the possibility to induce a band gap controlled by chemical doping ${ }^{5}$ or by a perpendicular electric field. ${ }^{6-8}$ The standard FETs commonly work at high bias voltage where electron-phonon interactions play a major role in the electronic transport. As a consequence, better knowledge of the electronic interactions in BLG is of importance for optimizing graphene-based nanoelectronics devices.

The electronic mobility of BLG samples supported on $\mathrm{SiO}_{2}$ does not exceed $10000 \mathrm{~cm}^{2} \mathrm{~V}^{-1} \mathrm{~s}^{-1}$ due to a strong long-range scattering from charged impurities. ${ }^{9-11}$ In suspended ${ }^{12}$ or boron-nitride-supported ${ }^{13,14}$ BLG samples of higher mobility, the charged impurities scattering is much weaker and the shortrange scattering (potentially caused by point defects, neutral scatterers, or vacancies) is of importance. We consider here the low-temperature limit $\left(T_{0} \sim 4 \mathrm{~K}\right)$ where the electron-phonon coupling is negligible in the linear-response regime. ${ }^{15,16}$ With increasing bias voltage, the BLG differential conductivity in our samples initially goes up as a result of self-heating. ${ }^{17}$ At high bias voltages, electron-optical phonon coupling is relevant and considerably influences the electronic transport. In MLG samples, partial current saturation was reported and related to electron-optical phonon (e-op) coupling. ${ }^{18-21}$ This coupling was confirmed by investigating the phonon temperature by Raman spectroscopy. ${ }^{22-24}$ In supported samples, the population of the different optical phonon modes is difficult to quantify partly because both the intrinsic optical phonons of the graphene and those of the $\mathrm{SiO}_{2}$ surface can be involved.

In this work, we present electronic conductance and shot-noise measurements on several BLG samples. At high bias voltage, the decrease of both the conductance and the Fano factor are interpreted as signatures of electron-optical phonon coupling in BLG. The absence of current saturation is consistent with this coupling being weaker compared to that in MLG as calculated by Borysenko et al. ${ }^{25}$ Nevertheless, in this regime we may expect either the electron-phonon or electron-electron interactions to be strong enough for establishing a quasiequilibrium electron energy distribution, and consequently an estimate of the effective electronic temperature can directly be extracted from shot noise..$^{26,27}$ In this way, we find that the electronic temperature of our BLG samples can go up to $\sim 1200 \mathrm{~K}$ at a voltage of $0.75 \mathrm{~V}$, which confirms strong self-heating ${ }^{17}$ and which agrees with optical spectroscopy experiments. ${ }^{21}$ Finally, we make use of the electronic temperature to extract an approximate for the e-op interaction time $\left(\tau_{\text {e-op }}\right)$. We find that this inelastic interaction time decreases with bias voltage becoming close to the elastic interaction time (60 fs) at a voltage of $0.6 \mathrm{~V}$ in a 230-nm-long BLG sample.

\section{SAMPLE FABRICATION AND EXPERIMENTAL SETUP}

The BLG samples have been mechanically exfoliated from graphite by means of a semiconductor wafer dicing tape. A strongly doped Si substrate, separated by $d=250$ or $270 \mathrm{~nm}$ of $\mathrm{SiO}_{2}$ from the sample, was used as a back gate to tune the BLG charge density $n_{g}$. The leads were patterned using standard $e$-beam lithography techniques and a bilayer resist was employed to facilitate the liftoff. The samples were contacted using Ti/Al/Ti sandwich structures with thicknesses $10 \mathrm{~nm} / h / 5 \mathrm{~nm}$ where $h$ was varied over $50-70 \mathrm{~nm}(10 \mathrm{~nm}$ of $\mathrm{Ti}$ is the contact layer). Metal evaporation was made in an ultrahigh vacuum chamber $\left(10^{-10} \mathrm{mBar}\right)$ in order to obtain 
the highest contact transparency. ${ }^{28}$ Samples of various lengths $L$ from $230 \mathrm{~nm}$ up to $1 \mu \mathrm{m}$ and widths $W$ from 0.9 to $1.6 \mu \mathrm{m}$ were fabricated. All the data were measured using a two-lead configuration in a ${ }^{4} \mathrm{He}$ dewar at a bath temperature of $T_{0}=4.2 \mathrm{~K}$.

Differential ac conductivity was recorded at frequency $f=$ $32 \mathrm{~Hz}$ with a standard lock-in technique. The average Fano factor $F \equiv\left[S_{I}(I)-S_{I}(0)\right] / 2 e I$ was calculated by integrating the current spectral density $S_{I}$ over the frequency range $f=600-900 \mathrm{MHz}$. The noise generated by the sample was successively amplified by means of a home-made low-noise amplifier $^{29}$ at $4 \mathrm{~K}$ and room-temperature amplifiers by 16 and $70 \mathrm{~dB}$, respectively. The ${ }^{4} \mathrm{He}$ dewar was placed in a shielded room in order to protect noise measurements against external microwave radiation. After the two amplification stages, the noise was filtered with a band-pass filter (600-900 MHz) and rectified using a Schottky diode. ${ }^{4}$ At low noise power $(<10 \mathrm{~mW})$, the dc voltage measured after the diode is directly proportional to the noise power. The noise calibration was done by measuring the shot noise of a tunnel junction, which is known to have a Fano factor $\mathcal{F}=1$ (see Ref. 30). A microwave switch allowed us to use the same amplification channel for measuring the noise of the graphene sample and the tunnel junction. $^{4}$

\section{INTRODUCTION TO SHOT NOISE}

In mesoscopic devices, shot noise originates from the granular nature of charge carriers. Shot noise contains information on the electronic transport properties that cannot be obtained by simple conductance measurements. ${ }^{30}$ Moreover, shot noise can be used to probe the electronic temperature of mesoscopic systems and it is even possible to use this effect as a primary thermometer. $^{31}$ At the zero-frequency limit, shot noise is given by the correlation function of current fluctuations $\delta I(t)$ : $S_{I}=\int d t\langle\delta I(t) \delta I(0)\rangle$. Typically, the strength of shot noise is characterized by the Fano factor, defined as $\mathcal{F}=S_{I} / 2 e\langle I\rangle$. The measured average Fano factor $F$ is approximately related to the true Fano factor $\mathcal{F}$ via the Khlus formula: ${ }^{32}$

$$
F \approx\left(\operatorname{coth} \frac{e V}{2 k_{B} T}-\frac{2 k_{B} T}{e V}\right) \mathcal{F} .
$$

Note that when $e|V| \gg k_{B} T$, the measured $F$ is close to the true Fano factor $(F \approx \mathcal{F})$. The Fano factor is different depending on the considered mesoscopic sample. For example, it is 1 for a low-transparency tunnel junction, $1 / 3$ for a diffusive metallic wire, ${ }^{33,34} 1 / 4$ for a chaotic cavity, ${ }^{35-38}$ and 0 for a ballistic sample. ${ }^{39,40}$ The shot-noise measurements were used to measure the effective charge of $e / 3$ in the fractional quantum Hall regime ${ }^{41,42}$ and $2 e$ in a $\mathrm{SN}$ junction. ${ }^{43,44}$ It could also be used to study many-body interactions and spin related phenomena. ${ }^{45,46}$ Shot noise has turned out to be very useful in the studies of graphene. ${ }^{4,47}$ For ballistic bilayer graphene, theoretical calculations give either $\frac{1}{3}$ (Ref. 48) or $1-\frac{2}{\pi},{ }^{49}$ i.e., very close to what has been observed at the charge neutrality point (CNP) in monolayers ${ }^{4,47}$ and to the value $\frac{1}{3}$ for diffusive conductors without inelastic interactions. ${ }^{30} \mathrm{By}$ reducing the width of MLG sheets down to nanoribbons, a strong suppression of shot noise has been observed consistent with inelastic hopping in these disordered systems. ${ }^{50}$
If local temperature $T(x)$ is well defined (quasiequilibrium) at every position $x$ along the length of the graphene sheet, then it can be shown using theory for diffusive conductors that (for $\left.e|V| \gg k_{B} T_{0}\right)^{51,52}$

$$
\mathcal{F} \approx \frac{2 k_{B} T_{e}}{e V},
$$

where $T_{e} \equiv(1 / L) \int_{0}^{L} d x T(x)$ is the average temperature. ${ }^{53}$ The electron-electron $(e-e)$ interactions may lead to an increase of $\mathcal{F}$ over the value $\frac{1}{3} .^{51}$ This happens if the power carried away via e-op coupling is small, such that all the injected power goes to the leads. Then $k_{B} T_{e} \approx \frac{\sqrt{3}}{8} e V$, i.e., $\mathcal{F}=\frac{\sqrt{3}}{4}>\frac{1}{3}$, the "hot electron" value. ${ }^{30}$ Now, if heat leaks out from the system also via electron-optical phonon (e-op) coupling, then $T_{e}$ is reduced and hence $\mathcal{F}$ decreases. Thus there are two opposite tendencies for $\mathcal{F}(V)$, but at very large bias the e-op coupling will dominate and reduce the noise.

\section{EXPERIMENTAL RESULTS}

In this paper, we focus on the results obtained on three different BLG samples. The first one is a $0.23-\mu \mathrm{m}-$ long and 1.1- $\mu \mathrm{m}$-wide BLG sample shown in the lower inset of Fig. 1. This sample located in between terminals $\mathrm{H} 3$ and $\mathrm{H} 4$ is named H34 in the following. We report also conductance measurements on two other samples of different length $(0.35$ and $0.95 \mu \mathrm{m}$ ), which have been built from the same BLG sheet (see lower left inset of Fig. 3). Experimental findings reported on these samples are qualitatively similar to those found on all the other measured BLG samples.

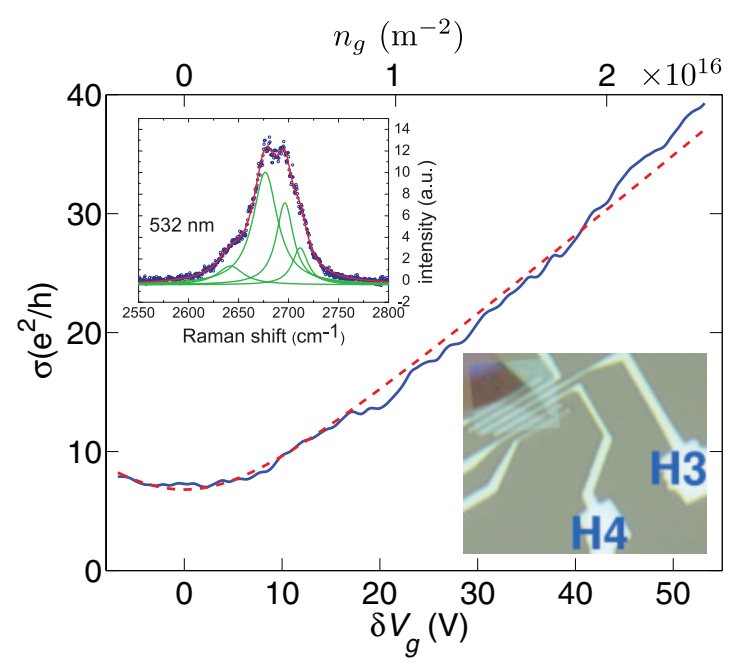

FIG. 1. (Color online) Conductivity of sample H34 as a function of the normalized gate voltage $\delta V_{g} \equiv V_{g}-V_{g, \min }$, where $V_{g, \min }=$ $-23 V$ is the value of $V_{g}$ at the charge neutrality point (CNP). The solid line depicts the experimental data. The dashed line is a fit by using $\sigma=e \mu \sqrt{n_{g}^{2}+n_{*}^{2}}$, with the residual density $n_{*}=4.7 \times 10^{15} \mathrm{~m}^{-2}$ and the mobility $\mu=3600 \mathrm{~cm}^{2} \mathrm{~V}^{-1} \mathrm{~s}^{-1}$ (equivalent to an impurity density $n_{\text {imp }}=3.5 \times 10^{15} \mathrm{~m}^{-2}$ ). Optical interferogram of sample H34 located between leads $\mathrm{H} 3$ and $\mathrm{H} 4$ (of dimension $0.23 \times 1.1 \mu \mathrm{m}^{2}$ ) is illustrated in the right inset. To make the sheet more visible, digital contrast enhancement has been applied. The upper inset displays our typical bilayer Raman spectrum with four lines fitted. 


\section{A. Electronic mobility}

In this section, we extract the electronic mobility of sample H34, which will be used later in Sec. V to calculate the theoretical BLG conductivity. Figure 1 presents the gate voltage dependence of the zero-bias conductivity $\sigma=$ $\left.(L / W)(d I / d V)\right|_{V=0}$ of sample $\mathrm{H} 34$ at $4.2 \mathrm{~K}$. The conductivity is minimal and equal to $6.9 e^{2} / h$ at the so-called charge neutrality point at $V_{g}=V_{g \text {, min }}=-23 \mathrm{~V}$. This sample is thus $n$ doped in the absence of a gate voltage. The gate voltage axis in Fig. 1 has been renormalized such that $\delta V_{g}=V_{g}-V_{g \text {,min }}$. In other BLG samples, we commonly observed an asymmetry between the zero-bias conductance in the $p$ and $n$ regions, the conductivity being lower in the $p$ region. This asymmetry is more pronounced in short samples and probably originates from the $n$ doping by the leads ${ }^{54}$ and the ensuing formation of a $p$ - $n$ junction close to the contact-graphene interface when BLG is $p$ doped $^{17,54-58}\left(\delta V_{g}<0\right)$. The $p$-doped region has not been measured for sample $\mathrm{H} 34$ because of a strong initial $n$ doping by impurities and the leads. The dashed line in Fig. 1 corresponds to the zero-bias conductance fit using the (zero-temperature) empirical relation $\sigma=e \mu \sqrt{n_{g}^{2}+n_{*}^{2}}$, where $n_{*}$ is the "residual" density and $\mu$ is the mobility. In the parallel plate model, the charge density $n_{g}$ is related to the gate voltage $\delta V_{g}$ via $n_{g}=C_{g} \delta V_{g} / e$, with $C_{g}=\epsilon_{r} \epsilon_{0} / d$ the gate capacitance per area. In short samples, the screening of the electric field by the leads decreases the effective gate capacitance. This modification can be accounted for in the parallel-plate model by replacing $\epsilon_{r}=3.9$ by a lower effective dielectric constant $\epsilon_{\text {eff }}$. By solving the Poisson equation, ${ }^{59}$ we find $\epsilon_{\text {eff }}=0.55 \times \epsilon_{r}=2.1$ and $C_{g}=85 \mathrm{aF} / \mu \mathrm{m}^{2}$.

Using this gate capacitance value, we extract the mobility $\mu=3600 \mathrm{~cm}^{2} \mathrm{~V}^{-1} \mathrm{~s}^{-1}$ and the residual density $n_{*}=4.7 \times$ $10^{15} \mathrm{~m}^{-2}$. In BLG, the impurity density $n_{\text {imp }}$ is related to the mobility $\mu$ via the relation ${ }^{17} n_{\mathrm{imp}}=8 e /\left(\pi^{2} \hbar \mu\right)$, which gives $n_{\text {imp }}=3.5 \times 10^{15} \mathrm{~m}^{-2}$.

\section{B. Bias voltage dependence of BLG conductivity}

The bias voltage dependence of the differential conductivity $\sigma(V)=(L / W)(d I / d V)$ of sample $\mathrm{H} 34$ is shown in Fig. 2 for three different gate voltages. At low bias, the conductivity increases linearly with voltage, leading to a superlinear current-voltage $[I(V)]$ characteristic. This nonlinearity is specific to bilayer graphene and does not show up in monolayer graphene (MLG). It has been explained in Ref. 17 by selfheating, though other contributions cannot be excluded in the case of this sample. Indeed, the conductivity in BLG is more sensitive to temperature than in MLG due to a higher density of states. ${ }^{9}$ Above $0.1 \mathrm{~V}$, the BLG conductivity grows slower and slower as the voltage increases, and reaches a maximum at $0.2 \mathrm{~V} \ldots 0.75 \mathrm{~V}$. Above this voltage the conductivity begins to slowly decrease.

We also investigated the transport properties of two BLG samples of different length (350 and $950 \mathrm{~nm}$ ) made from the same BLG sheet. ${ }^{17}$ As shown in Fig. 3 , at low bias and for the same charge density, the conductivity does not depend on the length $L$ of the sample, showing the same initial increase with voltage. However, at higher bias, the conductivity of the short sample reaches a maximum and then decreases faster than in

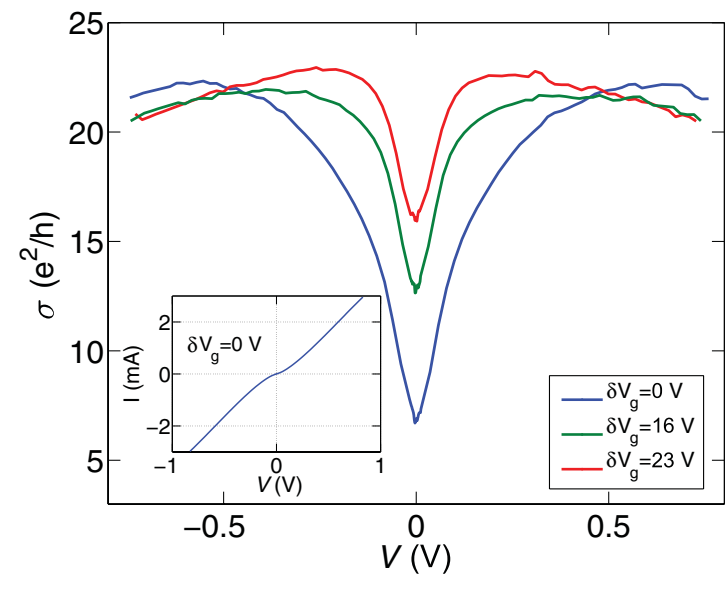

FIG. 2. (Color online) Conductivity of sample H34 as a function of voltage measured at three different gate voltages at $T=4.2 \mathrm{~K}$. Inset: $I(V)$ curve of sample $\mathrm{H} 34$ at CNP.

the long sample. We define $V_{d}$ as the voltage above which the conductivities of the short and long samples deviate.

In order to explain these experimental results, we now focus on the backscattering induced by interactions between electrons and optical phonons (op). Note that extrinsic surface phonons modes of the $\mathrm{SiO}_{2}$ substrate are here relevant and may be the primary source of energy relaxation. ${ }^{60,61}$ In a simple model, we assume that the electrons are accelerated by the external electric field $\mathcal{E} \equiv V / L$, so the energy gained by the electron after a distance $x$ is $e|\mathcal{E}| x$. When this energy reaches the characteristic optical phonon energy $\hbar \Omega$, electrons can transmit their energy to optical phonons (op). Assuming an instantaneous energy exchange, the "threshold" mean-free path for the onset of scattering is thus given by ${ }^{62} x \sim \hbar \Omega / e \mathcal{E}$. At the same bias voltage, the electric field is higher in the short sample and, consequently, the threshold mean free path is shorter. This explains why the conductivity of the short sample is lower than that of the long sample. At low bias

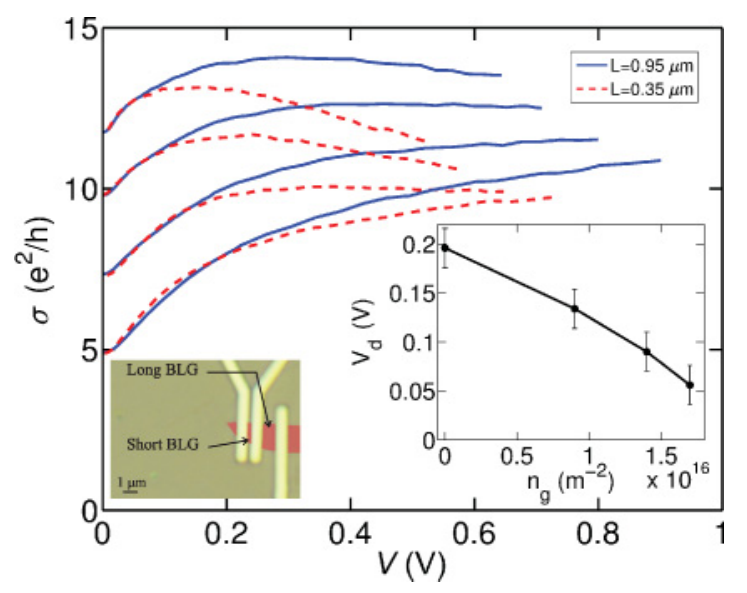

FIG. 3. (Color online) Conductivity vs voltage for two BLG samples of different lengths (350 and $950 \mathrm{~nm}$ ) at four different charge densities (from bottom to top curve pairs: $n_{g}=0,0.9,1.4$, and $1.75 \times$ $10^{16} \mathrm{~m}^{-2}$ ). Left inset: optical image of the short and long samples made from the same BLG flake, which is colored in red (dark gray) to enhance its visibility. Right inset: voltage $V_{d}$ at which the conductivities of the short and long samples start to deviate. 


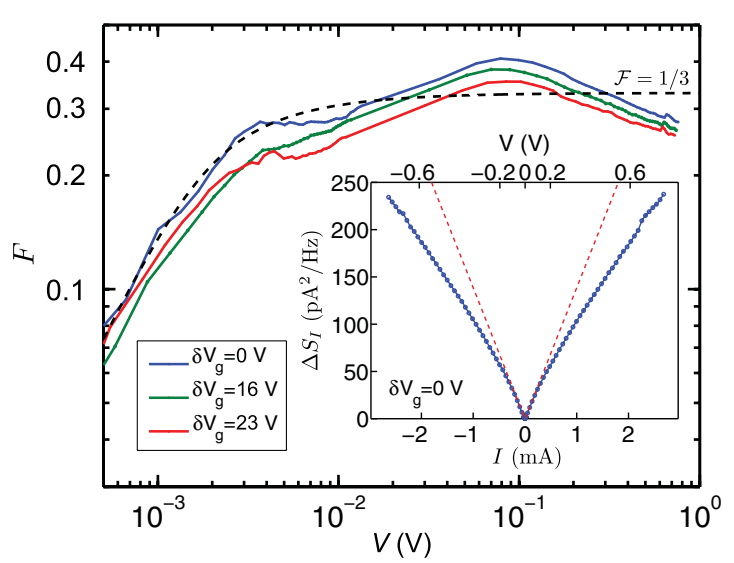

FIG. 4. (Color online) Excess noise Fano factor $F=\left[S_{I}(I)-\right.$ $\left.S_{I}(0)\right] /(2 e I)$ vs $V$ at three values of $\delta V_{g}$ indicated in the figure. The dashed curve is calculated using Eq. (1) for $\mathcal{F}=1 / 3$. Inset: $\Delta S_{I}=S_{I}(I)-S_{I}(0)$ as a function of the current $I$ at CNP. The dashed lines depict the theoretical values of $\Delta S_{I}(I)=\mathcal{F} 2 e I$ with a constant Fano factor $\mathcal{F}=\sqrt{3} / 4$, making clear the drop of $\mathcal{F}$ above $V \sim 0.2 \mathrm{~V}$. The nonlinearity of the voltage axis (upper axis) comes from the nonlinear $I(V)$ curve.

voltages, the fact that the conductivities of both samples are almost identical is in agreement with a self-heating effect ${ }^{17}$ in absence of electron-phonon coupling. The voltage $V_{d}$ is lowered when the charge density increases (inset of Fig. 3), suggesting an enhancement of backscattering from phonons far from the CNP.

\section{Shot noise in BLG}

Additional information to electronic conductivity measurements was obtained from shot noise in BLG. In particular, an effective electronic temperature was directly obtained from shot noise, which is analyzed in Sec. V B to extract an estimate for the e-op interaction time. Figure 4 presents the voltage dependence of the average Fano factor $F$ for the same gate voltages as for the conductivity curves in Fig. 2. At low bias voltages $V \lesssim 2 \mathrm{mV}, F$ increases linearly with voltage as expected from the Khlus formula (1) when $e V \ll 2 k_{B} T$ : $F \approx \mathcal{F} e V / 6 k_{B} T$. By fitting the noise at low bias with the Khlus formula with a fixed temperature $T=4.2 \mathrm{~K}$, we find that $\mathcal{F}$ is close to $1 / 3$ at the $\mathrm{CNP}$ as expected for a diffusive sample, ${ }^{30}$ and it is slightly lower far from the CNP, which may be related to disorder ${ }^{63-66}\left(\mathcal{F} \sim 0.3\right.$ at $\delta V_{g}=16$ and $\left.23 \mathrm{~V}\right)$. At bias voltages above $20 \mathrm{mV}$, the average Fano factor is weakly dependent on gate voltage, and $F$ first goes higher than predicted from the Khlus formula. We interpret this as a signature of a hot-electron regime. ${ }^{33}$ Above $0.1 \mathrm{~V}, F$ decays and goes to a value of $\sim 0.26$ at $V=0.75 \mathrm{~V}$. We assign the decrease of the Fano factor at high bias voltages to the coupling between electrons and optical phonons.

\section{ELECTRON-OPTICAL PHONON SCATTERING TIME}

The theoretical conductivity for BLG is a function of the chemical potential $E_{F}$, the average electronic temperature $T_{e}$, and the electron scattering time $\tau$, which is independent of energy for screened Coulomb impurities. ${ }^{17}$ Introducing again the phenomenological residual density $n_{*}$, we may write the conductivity as

$$
\sigma\left(\tau, E_{F}, T_{e}\right)=e \mu \sqrt{\left(n_{e}+n_{h}\right)^{2}+n_{*}^{2}},
$$

with $n_{e}=\left\{\gamma_{1} k_{B} T_{e} /\left[\pi\left(\hbar v_{0}\right)^{2}\right]\right\} \ln \left(1+e^{E_{F} / k_{B} T_{e}}\right)$ and $n_{h}=$ $\left\{\gamma_{1} k_{B} T_{e} /\left[\pi\left(\hbar v_{0}\right)^{2}\right]\right\} \ln \left(1+e^{-E_{F} / k_{B} T_{e}}\right)$ the electron and hole concentrations, respectively. The chemical potential $E_{F}$ is related to the charge density $n_{g}=n_{e}-n_{h}$ via the relation $E_{F}=\left[\pi\left(\hbar v_{0}\right)^{2} / \gamma_{1}\right] n_{g}$. We assume that electron and hole mobilities are identical ${ }^{17}$ and given by $\mu=\left(2 e v_{0}^{2} / \gamma_{1}\right) \tau$. Using Matthiessen's rule, we simply replace the scattering rate $\tau^{-1}$ by the sum of two contributions: $\tau^{-1}=\tau_{\mathrm{imp}}^{-1}+\tau_{\mathrm{e}-\mathrm{op}}^{-1}$, coming from charged impurity and electron-phonon scattering, respectively. At zero bias, charged impurity scattering dominates, ${ }^{15,16}$ so $\tau(0 \mathrm{~V})=\tau_{\text {imp. }}$. Using the zero bias mobility $\mu=3600 \mathrm{~cm}^{2} \mathrm{~V}^{-1} \mathrm{~s}^{-1}$, we deduce $\tau(0 \mathrm{~V})=60 \mathrm{fs}$. We assume next that $\tau_{\text {imp }}$ does not depend on voltage.

In the following, we first consider only scattering from charged impurities, neglecting inelastic processes, i.e., $\tau=$

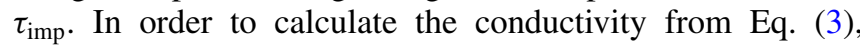
the electronic temperature $\left(T_{e}\right)$ has to be known. In the next section, $T_{e}$ is extracted at low bias by solving the heat diffusion equation in absence of electron-phonon coupling and at high bias from shot-noise measurements (in presence of e-op coupling). We note again that both the optical phonons of the graphene itself and those of the substrate can be relevant sources of e-op scattering, ${ }^{15,60,61}$ but we do not need to specify the type of the phonons in our phenomenological analysis.

\section{A. Electronic temperature}

As shown in Sec. IV B, at low bias voltage the electronphonon interactions can be neglected. Under this condition, assuming that the electron energy distribution function is at quasiequilibrium, the electronic temperature at the position $x$ along the BLG sheet is given by solving the heat diffusion equation, using the Wiedemann-Franz law. In the limit where $T_{e} \gg T_{0}=4 \mathrm{~K}$, it yields

$$
T(x)=\sqrt{(x / L)(1-x / L) / \mathcal{L}} V,
$$

where $\mathcal{L}=\pi^{2} k_{B}^{2} /\left(3 e^{2}\right)$ is the Lorenz number. The average temperature $T_{e}=\frac{\sqrt{3}}{8} e V / k_{B}$ increases linearly with $V$ and it goes up to $250 \mathrm{~K}$ at $0.1 \mathrm{~V}$ (see dashed line in Fig. 5) .

Above $\sim 0.1 \mathrm{~V}$, electrons interact with optical phonons, and thus the average temperature calculated from Eq. (4) is an overestimate. In this inelastic interaction regime, the electronic temperature can be extracted from the Fano factor using Eq. (2). We find $T_{e} \sim 1200 \mathrm{~K}$ at $0.75 \mathrm{~V}$, which is much lower than the temperature of $2300 \mathrm{~K}$ we would observe without energy relaxation to phonons (dashed line in Fig. 5). Note that the electronic temperature does not depend much on gate voltage at high bias. This can be understood from the fact that the broadening of the electron energy distribution $\left(\sim k_{B} T\right)$ is much larger than the chemical potential $E_{F}$.

At the CNP and for a electrical power density of $500 \mathrm{~kW} / \mathrm{cm}^{2}$, the electronic temperature goes up to $1000 \mathrm{~K}$, which is of a similar magnitude with the phonon temperatures found in monolayer graphene by infrared spectroscopy ${ }^{21,22}$ and Raman spectroscopy. ${ }^{23,24}$ 


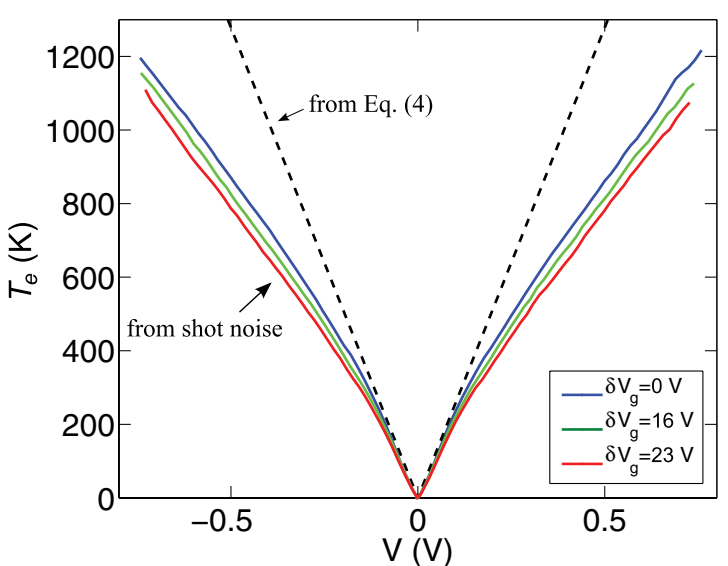

FIG. 5. (Color online) The solid lines are the electronic temperature extracted from the Fano factor $\left[T_{e}=e V \mathcal{F} /\left(2 k_{B}\right)\right]$ as a function of voltage at three different gate voltages given in the inset. The dashed line depicts the gate voltage independent average temperature calculated using Eq. (4) in absence of e-op coupling.

\section{B. Theoretical conductivity and electron-optical phonon scattering time}

The dotted lines shown in Fig. 6 depict the theoretical conductivities at low bias as a function of voltage calculated without any free parameters by using Eq. (3) and the average temperature $T_{e}$ from Eq. (4). The theoretical conductivity does not change much at low bias and increases at higher bias linearly with voltage. This leads to a low bias conductivity "plateau" whose width grows with increasing the chemical potential $E_{F}$. This conductivity plateau has not been observed in any of our measured BLG samples as discussed in Ref. 17.

At high bias voltages $(V>0.2 \mathrm{~V})$, by using the temperature extracted from the Fano factor, we find that the theoretical conductivity given by Eq. (3) increases linearly with increasing $V$ and is almost always above the experimental conductivity

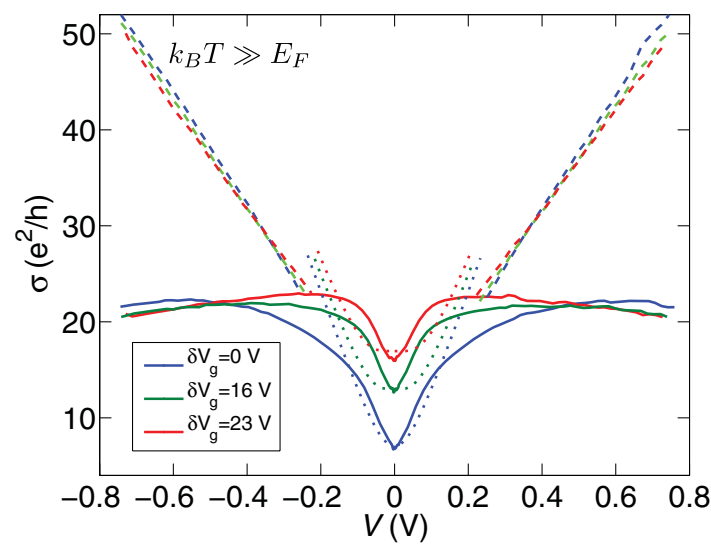

FIG. 6. (Color online) The solid lines depict the measured conductivity as a function of voltage at three different gate voltages. The dotted and dashed lines are the theoretical conductivities [Eq. (3)] calculated using the temperature obtained from Eq. (4) and from the Fano factor, respectively.

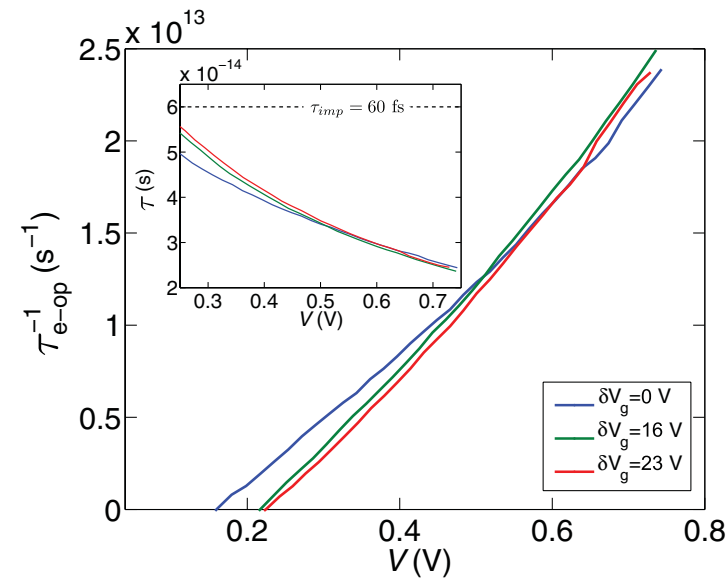

FIG. 7. (Color online) Electron-phonon scattering rate $\tau_{\mathrm{e}-\mathrm{op}}^{-1}$ as a function of voltage in sample H34. Inset: voltage dependence of the total scattering time $\tau=\left(\tau_{\mathrm{imp}}^{-1}+\tau_{\mathrm{e}-\mathrm{op}}^{-1}\right)^{-1}$.

(dashed lines in Fig. 6). As a result, the discrepancy between the theoretical and experimental conductivities increases with increasing $V$. This was to be expected as we have only considered so far charged impurity scattering and ignored e-op phonon interactions.

We next fit the experimental conductivity with Eq. (3) by adjusting the interaction time $\tau_{\mathrm{e} \text {-op }}$. Note that the temperature extracted from the Fano factor takes into account e-op interactions and thus is still valid. At $V \sim 0.2 \mathrm{~V}$, the theoretical and experimental conductivities are close, resulting in a large uncertainty for $\tau_{\mathrm{e}-\mathrm{op}}$. We find that the scattering rate $\tau_{\mathrm{e} \text {-op }}^{-1}$ does not depend much on gate voltage (Fig. 7 ). This can again be understood from the fact that $k_{B} T \gg E_{F}$. The rate $\tau_{\text {e-op }}^{-1}$ is very low $\left(<0.2 \times 10^{13} \mathrm{~s}^{-1}\right)$ at voltages below $0.2 \mathrm{~V}$ and charged impurity scattering dominates. Above $V=0.2 \mathrm{~V}, \tau_{\mathrm{e}-\mathrm{op}}^{-1}$ increases almost linearly with increasing the bias voltage, as calculated for MLG. ${ }^{67}$ At $V=0.75 \mathrm{~V}$, the inelastic rate is $\sim 1 / 40 \mathrm{fs}^{-1}$ which is close to $\tau_{\mathrm{imp}}^{-1}=1 / 60 \mathrm{fs}^{-1}$. The increase of the inelastic scattering rate $\tau_{\mathrm{e}-\mathrm{op}}^{-1}$ can be explained by the presence of more phase space for the e-op scattering.

\section{CONCLUSION}

In conclusion, we have investigated conductance and shot noise in several BLG samples at a bath temperature of $4.2 \mathrm{~K}$. At low bias voltage, the increase of the differential conductivity with voltage is attributed to self-heating. In this regime, the scattering time is limited by charged impurity scattering. At high bias voltage, decrease in the conductance indicates electron-optical phonon scattering, which is also confirmed by reduction of shot noise. The drop of the conductivity is more pronounced in short samples, suggesting a larger e-op scattering rate. This agrees with the expectation that the threshold mean-free-path for e-op coupling is directly proportional to the sample length. At high bias voltage, the electronic temperature $T_{e}$ in the BLG sheet is deduced from shot-noise measurements. The electronic temperature goes up 
to $\sim 1200 \mathrm{~K}$ at a voltage of $0.75 \mathrm{~V}$. From the temperature $T_{e}$, we estimated the e-op scattering time and found that it decreases with increasing voltage. In a 230-nm-long BLG sample, we find that $\tau_{\mathrm{e} \text {-op }}$ goes below the elastic-scattering time $\tau_{\text {imp }}=60 \mathrm{fs}$ at a voltage of $0.6 \mathrm{~V}$. The e-op coupling is thus manifested at the standard working points of graphene-based FETs. Extended work on suspended graphene samples is of interest in order to suppress the relaxation channel to $\mathrm{SiO}_{2}$ surface phonons.

\section{ACKNOWLEDGMENTS}

This work was supported by the Academy of Finland, Grant No. EU-FP7-NMP-246026, the European Science Foundation (ESF) under EUROCORES Programme EuroGraphene, and the EU project MMM@HPC FP7-261594. R.D.'s Shared Research Group SRG 1-33 received financial support by the Karlsruhe Institute of Technology within the framework of the German Excellence Initiative.
*Present address: Department of Physics, P.O. Box 3000, FIN-90014 University of Oulu, Finland.

${ }^{1}$ A. H. Castro Neto, F. Guinea, N. M. R. Peres, K. S. Novoselov, and A. K. Geim, Rev. Mod. Phys. 81, 109 (2009).

${ }^{2}$ K. I. Bolotin, K. J. Sikes, Z. Jiang, M. Klima, G. Fudenberg, J. Hone, P. Kim, and H. L. Stormer, Solid State Commun. 146, 351 (2008).

${ }^{3}$ F. Miao, S. Wijeratne, Y. Zhang, U. C. Coskun, W. Bao, and C. N. Lau, Science 317, 1530 (2007).

${ }^{4}$ R. Danneau, F. Wu, M. F. Craciun, S. Russo, M. Y. Tomi, J. Salmilehto, A. F. Morpurgo, and P. J. Hakonen, Phys. Rev. Lett. 100, 196802 (2008); J. Low Temp. Phys. 153, 374 (2008); Solid State Commun. 149, 1050 (2009).

${ }^{5}$ T. Otah, A. Bostwick, T. Seyller, K. Horn, and E. Rotenberg, Science 313, 951 (2006).

${ }^{6}$ F. Xia, D. B. Farmer, Y.-M. Lin, and P. Avouris, Nano Lett. 10, 715 (2010).

${ }^{7}$ L. Jing, J. Velasco Jr., P. Kratz, G. Liu, W. Bao, M. Bockrath, and C. N. Lau, Nano Lett. 10, 4000 (2010).

${ }^{8}$ J. B. Oostinga, H. B. Heersche, X. Liu, A. F. Morpurgo, and L. M. K. Vandersypen, Nat. Mater. 7, 151 (2008).

${ }^{9}$ S. Adam and S. Das Sarma, Phys. Rev. B 77, 115436 (2008).

${ }^{10}$ S. V. Morozov, K. S. Novoselov, M. I. Katsnelson, F. Schedin, D. C. Elias, J. A. Jaszczak, and A. K. Geim, Phys. Rev. Lett. 100, 016602 (2008).

${ }^{11}$ S. Xiao, J.-H. Chen, S. Adam, E. D. Williams, and M. S. Fuhrer, Phys. Rev. B 82, 041406 (2010).

${ }^{12}$ B. E. Feldman, J. Martin, and A. Yacoby, Nat. Phys. 5, 889 (2009).

${ }^{13}$ C. R. Dean, A. F. Young, I. Meric, C. Lee, L. Wang, S. Sorgenfrei, K. Watanabe, T. Taniguchi, P. Kim, K. L. Shepard, and J. Hone, Nat. Nanotechnol. 5, 722 (2010).

${ }^{14}$ S. Das Sarma and E. H. Hwang, Phys. Rev. B 83, 121405(R) (2011).

${ }^{15}$ J. K. Viljas and T. T. Heikkilä, Phys. Rev. B 81, 245404 (2010).

${ }^{16}$ S. Adam and M. D. Stiles, Phys. Rev. B 82, 075423 (2010).

${ }^{17}$ J. K. Viljas, A. Fay, M. Wiesner, and P. J. Hakonen, Phys. Rev. B 83, 205421 (2011).

${ }^{18}$ I. Meric, M. Y. Han, A. F. Young, B. Ozyilmaz, Ph. Kim, and K. L. Shepard, Nat. Nanotechnol. 3, 654 (2008).

${ }^{19}$ A. Barreiro, M. Lazzeri, J. Moser, F. Mauri, and A. Bachtold, Phys. Rev. Lett. 103, 076601 (2009).

${ }^{20}$ V. Perebeinos and P. Avouris, Phys. Rev. B 81, 195442 (2010).

${ }^{21}$ M.-H. Bae, Z.-Y. Ong, D. Estrada, and E. Pop, Nano Lett. 10, 4787 (2011).

${ }^{22}$ M. Freitag, H.-Y. Chiu, M. Steiner, V. Perebeinos, and P. Avouris, Nat. Nanotech. 5, 497 (2010).
${ }^{23}$ D.-H. Chae, B. Krauss, K. von Klitzing, and J. H. Smet, Nano Lett. 10, 466 (2010).

${ }^{24}$ S. Berciaud, M. Y. Han, K. F. Mak, L. E. Brus, P. Kim, and Tony F. Heinz, Phys. Rev. Lett. 104, 227401 (2010).

${ }^{25}$ K. M. Borysenko, J. T. Mullen, X. Li, Y. G. Semenov, J. M. Zavada, M. B. Nardelli, and K. W. Kim, Phys. Rev. B 83, 161402(R) (2011).

${ }^{26}$ D. F. Santavicca, J. D. Chudow, D. E. Prober, M. S. Purewal, and P. Kim, Nano Lett. 10, 4538 (2010).

${ }^{27}$ F. Wu, P. Virtanen, S. Andresen, B. Plaçais, and P. J. Hakonen, App. Phys. Lett. 97, 262115 (2010).

${ }^{28}$ We have measured maximum contact resistances on the order of $20 \Omega \mu \mathrm{m}^{2}$.

${ }^{29}$ L. Roschier and P. Hakonen, Cryogenics 44, 783 (2004).

${ }^{30}$ Ya. M. Blanter and M. Büttiker, Phys. Rep. 336, 1 (2000).

${ }^{31}$ L. Spietz, K. W. Lehnert, I. Siddiqi, and R. J. Schoelkopf, Science 300, 1909 (2003).

${ }^{32}$ V. A. Khlus, Sov. Phys. JETP 66, 1243 (1987) [Zh. Eksp. Teor. Fiz. 93, 2179 (1987)].

${ }^{33}$ A. H. Steinbach, J. M. Martinis, and M. H. Devoret, Phys. Rev. Lett. 76, 3806 (1996).

${ }^{34}$ M. Henny, S. Oberholzer, C. Strunk, and C. Schönenberger, Phys. Rev. B 59, 2871 (1999).

${ }^{35}$ P. W. Brouwer and C. W. J. Beenakker, J. Math. Phys. 37, 4904 (1996).

${ }^{36}$ S. Oberholzer, E. V. Sukhorukov, C. Strunk, C. Schönenberger, T. Heinzel, and M. Holland, Phys. Rev. Lett. 86, 2114 (2001).

${ }^{37}$ S. Oberholzer, E. V. Sukhorukov, and C. Schönenberger, Nature (London) 415, 765 (2002).

${ }^{38}$ P. G. Silvestrov, M. C. Goorden, and C. W. J. Beenakker, Phys. Rev. B 67, 241301(R) (2003).

${ }^{39}$ M. Reznikov, M. Heiblum, H. Shtrikman, and D. Mahalu, Phys. Rev. Lett. 75, 3340 (1995).

${ }^{40}$ A. Kumar, L. Saminadayar, D. C. Glattli, Y. Jin, and B. Etienne, Phys. Rev. Lett. 76, 2778 (1996).

${ }^{41}$ L. Saminadayar, D. C. Glattli, Y. Jin, and B. Etienne, Phys. Rev. Lett. 79, 2526 (1997).

${ }^{42}$ R. de-Picciotto, M. Reznikov, M. Heiblum, V. Umansky, G. Bunin, and D. Mahalu, Nature (London) 389, 162 (1997).

${ }^{43}$ X. Jehl, M. Sanquer, R. Calemczuk, and D. Mailly, Nature (London) 405, 50 (2000).

${ }^{44}$ A. A. Kozhevnikov, R. J. Schoelkopf, and D. E. Prober, Phys. Rev. Lett. 84, 3398 (2000).

${ }^{45}$ P. Roche, J. Ségala, D. C. Glattli, J. T. Nicholls, M. Pepper, A. C. Graham, K. J. Thomas, M. Y. Simmons, and D. A. Ritchie, Phys. Rev. Lett. 93, 116602 (2004). 
${ }^{46}$ L. DiCarlo, Y. Zhang, D. T. McClure, D. J. Reilly, C. M. Marcus, L. N. Pfeiffer, and K. W. West, Phys. Rev. Lett. 97, 036810 (2006).

${ }^{47}$ L. DiCarlo, J. R. Williams, Y. Zhang, D. T. McClure, and C. M. Marcus, Phys. Rev. Lett. 100, 156801 (2008).

${ }^{48}$ I. Snyman and C. W. J. Beenakker, Phys. Rev. B 75, 045322 (2007).

${ }^{49}$ M. I. Katsnelson, Eur. Phys. J. B 52, 151 (2006).

${ }^{50}$ R. Danneau, F. Wu, M. Y. Tomi, J. B. Oostinga, A. F. Morpurgo, and P. J. Hakonen, Phys. Rev. B 82, 161405(R) (2010).

${ }^{51}$ K. E. Nagaev, Phys. Rev. B 52, 4740 (1995).

${ }^{52}$ Y. Naveh, D. V. Averin, and K. K. Likharev, Phys. Rev. B 58, 15371 (1998).

${ }^{53}$ Using Eq. (2) for graphene close to the CNP is an approximation, because the derivation of the result requires a constant density of states.

${ }^{54}$ P. A. Khomyakov, A. A. Starikov, G. Brocks, and P. J. Kelly, Phys. Rev. B 82, 115437 (2010).

${ }^{55}$ B. Huard, N. Stander, J. A. Sulpizio, and D. Goldhaber-Gordon, Phys. Rev. B 78, 121402 (2008).

${ }^{56}$ S. Barraza-Lopez, M. Vanević, M. Kindermann, and M. Y. Chou, Phys. Rev. Lett. 104, 076807 (2010).
${ }^{57}$ E. J. H. Lee, K. Balasubramanian, R. T. Weitz, M. Burghard, and K. Kern, Nat. Nanotech. 3, 486 (2008).

${ }^{58}$ T. Mueller, F. Xia, M. Freitag, J. Tsang, and Ph. Avouris, Phys. Rev. B 79, 245430 (2009).

${ }^{59}$ The effective dielectric constant $\epsilon_{\text {eff }}$ used in the parallel plate model is chosen to give the same average charge density than calculated by solving analytically the Poisson equation in Ref. 17.

${ }^{60}$ J.-H. Chen, C. Jang, S. Xiao, M. Ishigami, and M. S. Fuhrer, Nat. Nanotechnol. 3, 206 (2008).

${ }^{61}$ S. Fratini and F. Guinea, Phys. Rev. B 77, 195415 (2008).

${ }^{62}$ Z. Yao, C. L. Kane, and C. Dekker, Phys. Rev. Lett. 84, 2941 (2000).

${ }^{63}$ P. San-Jose, E. Prada, and D. S. Golubev, Phys. Rev. B 76, 195445 (2007).

${ }^{64}$ J. Tworzydło, C. W. Groth, and C. W. J. Beenakker, Phys. Rev. B 78, 235438 (2008).

${ }^{65}$ C. H. Lewenkopf, E. R. Mucciolo, and A. H. Castro Neto, Phys. Rev. B 77, 081410(R) (2008).

${ }^{66}$ M. Titov, P. M. Ostrovsky, I. V. Gornyi, A. Schuessler, and A. D. Mirlin, Phys. Rev. Lett. 104, 076802 (2010).

${ }^{67}$ W.-K. Tse, E. H. Hwang, and S. D. Sarma, Appl. Phys. Lett. 93, 023128 (2008). 